\title{
Inversion Formulae on Permutations Avoiding 321
}

\section{Pingge Chen}

College of Mathematics and Econometrics Hunan University Changsha, P. R. China.

chenpingge@hnu.edu.cn
Zhousheng Mei

College of Mathematics and Econometrics

Hunan University

Changsha, P. R. China.

zhousheng@hnu.edu.cn

\author{
Suijie Wang* \\ College of Mathematics and Econometrics \\ Hunan University \\ Changsha, P. R. China. \\ wangsuijie@hnu.edu.cn
}

Submitted: July 29, 2015; Accepted: Nov 4, 2015; Published: Nov 13, 2015

\begin{abstract}
We will study the inversion statistic of 321-avoiding permutations, and obtain that the number of 321 -avoiding permutations on $[n]$ with $m$ inversions is given by

$$
\left|\mathcal{S}_{n, m}(321)\right|=\sum_{b \vdash m}\left(\begin{array}{c}
n-\frac{\Delta(b)}{2} \\
l(b)^{2}
\end{array}\right) .
$$

where the sum runs over all compositions $b=\left(b_{1}, b_{2}, \ldots, b_{k}\right)$ of $m$, i.e.,$$
m=b_{1}+b_{2}+\cdots+b_{k} \text { and } b_{i} \geqslant 1,
$$

$l(b)=k$ is the length of $b$, and $\Delta(b):=\left|b_{1}\right|+\left|b_{2}-b_{1}\right|+\cdots+\left|b_{k}-b_{k-1}\right|+\left|b_{k}\right|$. We obtain a new bijection from 321-avoiding permutations to Dyck paths which establishes a relation on inversion number of 321-avoiding permutations and valley height of Dyck paths.
\end{abstract}

Keywords: pattern avoidance; Catalan number; Dyck path; generating function

*Supported by NSFC 11401196 \& 11571097 


\section{Introduction}

Let $\mathcal{S}_{n}$ denote the permutation group on $[n]=\{1,2, \ldots, n\}$. Write $\sigma \in \mathcal{S}_{n}$ in the form $\sigma=\sigma_{1} \sigma_{2} \cdots \sigma_{n}$. For $m \leqslant n$, if $\sigma \in \mathcal{S}_{n}$ and $\pi=\pi_{1} \cdots \pi_{m} \in \mathcal{S}_{m}$, we say that $\sigma$ contains the pattern $\pi$ if there is an index subsequence $1 \leqslant i_{1}<i_{2}<\cdots<i_{m} \leqslant n$ such that $\sigma_{i_{j}}<\sigma_{i_{k}}$ iff $\pi_{j}<\pi_{k}$ for $1 \leqslant j, k \leqslant m$, that is, $\sigma$ has a subsequence which is order isomorphic to $\pi$. Otherwise, $\sigma$ avoids the pattern $\pi$, or say, $\sigma$ is $\pi$-avoiding. We denote by $\mathcal{S}_{n}(\pi)$ the set of all permutations $\sigma \in \mathcal{S}_{n}$ that are $\pi$-avoiding, i.e.,

$$
\mathcal{S}_{n}(\pi)=\left\{\sigma \in \mathcal{S}_{n} \mid \sigma \text { avoids the pattern } \pi\right\} .
$$

For example, the permutation 41253 avoids the pattern 321, but contains the pattern 132 since its subsequence 153 is order isomorphic to 132 , hence $41253 \in \mathcal{S}_{5}(321)$ and $41253 \notin \mathcal{S}_{5}(132)$.

In 1970's, Knuth $[12,13]$ obtained a well known result on permutations avoiding patterns, that is for any $\pi \in \mathcal{S}_{3}$,

$$
\left|\mathcal{S}_{n}(\pi)\right|=C_{n}=\frac{1}{n+1}\left(\begin{array}{c}
2 n \\
n
\end{array}\right)
$$

where $C_{n}$ is the $n$-th Catalan number which counts the number of Dyck paths of length $2 n$. In past decades, various articles considered the bijections between 321-avoiding permutations and Dyck paths, see [4, 7, 10, 11, 14, 15, 17, 18, 19, 21, 22].

In this paper, we will study the inversion distribution of 321-avoiding permutations. For $\sigma=\sigma_{1} \sigma_{2} \cdots \sigma_{n} \in \mathcal{S}_{n}(\pi)$, we define the inversion set $\operatorname{Inv}(\sigma)$ to be

$$
\operatorname{Inv}(\sigma)=\left\{\left(\sigma_{i}, \sigma_{j}\right) \mid i<j \text { and } \sigma_{i}>\sigma_{j}\right\}
$$

and denote by $\operatorname{inv}(\sigma)=\# \operatorname{Inv}(\sigma)$, called the inversion number of $\sigma$, where the hash sign denotes cardinality. The generating function $I_{n}(\pi, q)$ of the inversion numbers is

$$
I_{n}(\pi, q)=\sum_{\sigma \in S_{n}(\pi)} q^{\operatorname{inv}(\sigma)} .
$$

for $\sigma \in \mathcal{S}_{n}(\pi)$. This generating function was first introduced and explored in [8, 20] and some recurrence formulae have been obtained for $\pi \in S_{3}$ and $\pi \neq 321$. Conjecture 3.2 of [8] states that, for all $n \geqslant 1$,

$$
I_{n}(321, q)=I_{n-1}(321, q)+\sum_{i=0}^{n-2} q^{i+1} I_{i}(321, q) I_{n-i-1}(321, q) .
$$

Soon afterwards a bijective proof of the recursive formula (1) was obtained by Szu-En Cheng et al. [6]. There are some other works on inversions of restricted permutations, see $[1,3,5,9,15,16]$. In 2014, M. Barnabei, F. Bonetti, S. Elizalde and M. Silimbani [2] studied the distribution of descents and major indexes of 321-avoiding involutions. 
Motivated by $[2,6]$, in this paper we will study the inversion distribution of 321avoiding permutations. As the main result, we give an explicit formula counting the number of 321-avoiding permutations with the fixed inversion number. We also find a bijection between 321-avoiding permutations and Dyck paths, which is new to the best of our knowledge. From this bijection, we show that the inversion number of 321-avoiding permutations and the valley-sum of Dyck paths are equally distributed.

\section{Inversions of Permutations Avoiding 321}

For $1 \leqslant k \leqslant n$, let $\mathcal{S}_{n}^{k}(321)$ be the collection of 321 -avoiding permutations of $[n]$ and containing $12 \cdots k$ as a subsequence,

$$
\mathcal{S}_{n}^{k}(321)=\left\{\sigma \in \mathcal{S}_{n}(321) \mid \sigma^{-1}(1)<\sigma^{-1}(2)<\cdots<\sigma^{-1}(k)\right\} .
$$

More precisely, if $\sigma=\sigma_{1} \sigma_{2} \cdots \sigma_{n} \in \mathcal{S}_{n}^{k}(321)$ and $\sigma_{i_{1}}=1, \sigma_{i_{2}}=2, \ldots, \sigma_{i_{k}}=k$, then $i_{1}<i_{2}<\cdots<i_{k}$. Obviously, we have

$$
\mathcal{S}_{n}(321)=\mathcal{S}_{n}^{1}(321) \supseteq \mathcal{S}_{n}^{2}(321) \supseteq \cdots \supseteq \mathcal{S}_{n}^{n}(321)=\{\mathrm{id}\} .
$$

For $1 \leqslant k \leqslant n$, let $I_{n}^{k}(321, q)$ be the generating function defined by

$$
I_{n}^{k}(321, q)=\sum_{\sigma \in S_{n}^{k}(321)} q^{\operatorname{inv}(\sigma)} .
$$

Then we have $I_{n}(321, q)=I_{n}^{1}(321, q)$ and $I_{n}^{n}(321, q)=1$ for all $n \geqslant 1$.

Lemma 1. For $1 \leqslant k \leqslant n$, we have

$$
I_{n}^{k}(321, q)=I_{n}^{k+1}(321, q)+\sum_{i=1}^{k} q^{i} I_{n+i-k-1}^{i}(321, q)
$$

Proof. Given $\sigma \in \mathcal{S}_{n}^{k}(321)$ with $1 \leqslant k \leqslant n-1$, consider the position of $\sigma^{-1}(k+1)$. Assuming $\sigma^{-1}(0)=0$, we have either $\sigma^{-1}(k)<\sigma^{-1}(k+1)$, or $\sigma^{-1}(i)<\sigma^{-1}(k+1)<$ $\sigma^{-1}(i+1)$ for some $i \leqslant k-1$. (i): If $\sigma \in \mathcal{S}_{n}^{k}(321)$ and $\sigma^{-1}(k)<\sigma^{-1}(k+1)$, it follows that $\sigma \in \mathcal{S}_{n}^{k+1}(321)$. So this case contributes a term $I_{n}^{k+1}(321, q)$ to the generating function $I_{n}^{k}(321, q)$. (ii): If $\sigma \in \mathcal{S}_{n}^{k}(321)$ and $\sigma^{-1}(i)<\sigma^{-1}(k+1)<\sigma^{-1}(i+1)$ for some $i \leqslant k-1$, since $\sigma$ avoids the pattern 321, it forces that $\sigma^{-1}(j)>\sigma^{-1}(k+1)$ for all $j \geqslant k+2$. Otherwise, we have $\sigma^{-1}(j)<\sigma^{-1}(k+1)<\sigma^{-1}(i+1)$ which is obviously a contradiction. It implies that $\sigma=\sigma_{1} \sigma_{2} \cdots \sigma_{n}$ satisfies $\sigma_{1}=1, \sigma_{2}=2, \ldots, \sigma_{i}=i, \sigma_{i+1}=k+1$. Denote by $\bar{\sigma}=\sigma_{i+2} \sigma_{i+3} \cdots \sigma_{n}$. Then $\bar{\sigma}$ is a permutation of $\{i+1, \ldots, k, k+2, \ldots, n\}$ satisfying $\bar{\sigma}^{-1}(i+1)<\bar{\sigma}^{-1}(i+2)<\cdots<\bar{\sigma}^{-1}(k)$ and $\operatorname{inv}(\sigma)=k-i+\operatorname{inv}(\bar{\sigma})$. It implies that case (ii) contributes a term $q^{k-i} I_{n-i-1}^{k-i}(321, q)$ to $I_{n}^{k}(321, q)$ for $0 \leqslant i \leqslant k-1$. Changing the index $i$ to $k-i$, the proof will be complete by combining (i) and (ii). 
In the sequel, we always denote by $\bar{\delta}: \mathbb{R}^{2} \rightarrow\{0,1\}$ a function such that

$$
\bar{\delta}(u, v)=\left\{\begin{array}{lc}
0, & u=v \\
1, & \text { otherwise. }
\end{array}\right.
$$

In order to characterize the generating function $I_{n}(321, q)$ as a counting function of lattice points in a lattice polytope, we introduce the following lemma.

Lemma 2. Assuming $x_{0}=0$, for all $1 \leqslant t \leqslant n$, we have

$$
I_{n+1}^{1}(321, q)=\sum_{x_{1}=0}^{1} \sum_{x_{2}=x_{1}}^{2} \cdots \sum_{x_{t}=x_{t}-1}^{t} I_{n+1-x_{t}}^{t+1-x_{t}}(321, q) \prod_{i=1}^{t} q^{\bar{\delta}\left(x_{i}, x_{i-1}\right)\left(i+1-x_{i}\right)}
$$

Proof. The statement is true for $t=1$ by Lemma 1 . To use induction on $t$, suppose the above equality holds for $t$. From Lemma 1, we have

$$
I_{n+1-x_{t}}^{t+1-x_{t}}(321, q)=\sum_{x_{t+1}=x_{t}}^{t+1} I_{n+1-x_{t+1}}^{t+2-x_{t+1}}(321, q) q^{\bar{\delta}\left(x_{t+1}, x_{t}\right)\left(t+2-x_{t+1}\right)} .
$$

Using above formula to substitute the term $I_{n+1-x_{t}}^{t+1-x_{t}}(321, q)$ in the formula of this Lemma, we can easily conclude that the equality holds for the case $t+1$.

Let $\Omega_{n}$ be a convex lattice polytope defined by

$$
\Omega_{n}=\left\{\left(x_{1} \ldots, x_{n}\right) \in \mathbb{Z}^{n} \mid 0 \leqslant x_{1} \leqslant \cdots \leqslant x_{i} \leqslant i \text { for all } 1 \leqslant i \leqslant n\right\} .
$$

Recall that $I_{n+1}(321, q)=I_{n+1}^{1}(321, q)$ and $I_{n+1-x_{n}}^{n+1-x_{n}}(321, q)=1$. From above lemma by taking $t=n$ we can easily obtain

Proposition 3. Assuming $x_{0}=0$, we have

$$
I_{n+1}(321, q)=\sum_{x \in \Omega_{n}} \prod_{i=1}^{n} q^{\bar{\delta}\left(x_{i}, x_{i-1}\right)\left(i+1-x_{i}\right)}
$$

In the following we will give a more explicit interpretation about this formula. Let $\operatorname{inv}_{k}(\sigma)$ be the number of inversions of $\sigma$ whose first element is $k$, i.e,

$$
\operatorname{inv}_{k}(\sigma)=\#\{i \mid(k, i) \in \operatorname{Inv}(\sigma)\}
$$

It is obvious that $\operatorname{inv}_{k}(\sigma) \leqslant k-1$. From the definition of $I_{n+1}(321, q)$ and Proposition 3 , we have

$$
\sum_{\sigma \in \mathcal{S}_{n+1}(321)} q^{\operatorname{inv}(\sigma)}=\sum_{x \in \Omega_{n}} q^{\sum_{i=1}^{n} \bar{\delta}\left(x_{i}, x_{i-1}\right)\left(i+1-x_{i}\right)}
$$

Below we recursively define a map

$$
\varphi: \mathcal{S}_{n+1}(321) \rightarrow \Omega_{n}, \quad \varphi(\sigma)=\left(x_{1}, \ldots, x_{n}\right)=x,
$$




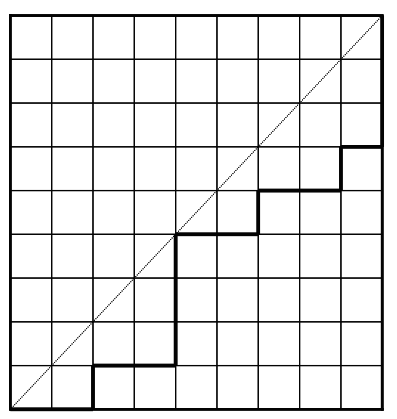

Figure 1: $\varphi: \sigma=312579468 \mapsto(0,0,2,0,1,0,2,0,3) \mapsto x=(0,1,1,4,4,5,5,6)$

such that $x_{1}=\operatorname{inv}_{2}(\sigma)$ and for $k \geqslant 2$,

$$
x_{k}=\left\{\begin{array}{cc}
x_{k-1}, & \text { if } \operatorname{inv}_{k+1}(\sigma)=0 \\
k+1-\operatorname{inv}_{k+1}(\sigma), & \text { otherwise }
\end{array}\right.
$$

Figure.1 shows an example, where the second vector is $\left(\operatorname{inv}_{1}(\sigma), \ldots, \operatorname{inv}_{9}(\sigma)\right)$.

Theorem 4. The map $\varphi$ defined above is a bijection. Moreover, if $\varphi(\sigma)=\left(x_{1}, \ldots, x_{n}\right)$, then

$$
\operatorname{inv}(\sigma)=\sum_{i=1}^{n} \bar{\delta}\left(x_{i}, x_{i-1}\right)\left(i+1-x_{i}\right) .
$$

Proof. We first show that $\varphi$ is well defined in the sense that if $x=\varphi(\sigma)$ then $x \in \Omega_{n}=$ $\left\{\left(x_{1}, \ldots, x_{n}\right) \in \mathbb{Z}^{n} \mid 0 \leqslant x_{1} \leqslant \cdots \leqslant x_{i} \leqslant i\right.$ for all $\left.1 \leqslant i \leqslant n\right\}$. We use induction on $i$. For $i=1$, it is obvious $x_{1}=i n v_{2}(\sigma) \leqslant 1$. Suppose $0 \leqslant x_{1} \leqslant \cdots \leqslant x_{i-1} \leqslant i-1$. If $\operatorname{inv}_{i+1}(\sigma)=0$, then $x_{i}=x_{i-1} \leqslant i$ by the induction hypothesis. If $\operatorname{inv}_{i+1}(\sigma) \neq 0$, then $x_{i}=i+1-\operatorname{inv}_{i+1}(\sigma) \leqslant i$. It remains to show that if $\operatorname{inv}_{i+1}(\sigma) \neq 0$, then $x_{i-1} \leqslant x_{i}$. Let $k \leqslant i$ be maximal such that $\operatorname{inv}_{k}(\sigma) \neq 0$, i.e., $\operatorname{inv}_{k+1}(\sigma)=\cdots=\operatorname{inv}_{i}(\sigma)=0$. It follows that there exists an inversion $(k, l) \in \operatorname{Inv}(\sigma)$. Since $\sigma$ is 321-avoiding, we have $\sigma^{-1}(k)<\sigma^{-1}(i+1)$, otherwise $(i+1, k, l)$ is a subsequence of $\sigma$ and of type 321. Hence we obtain $\operatorname{inv}_{i+1}(\sigma) \leqslant \operatorname{inv}_{k}(\sigma)+i-k$, and

$$
x_{i}=i+1-\operatorname{inv}_{i+1}(\sigma) \geqslant k+1-\operatorname{inv}_{k}(\sigma)=x_{k-1}+1>x_{k-1} .
$$

Note that $\operatorname{inv}_{k+1}(\sigma)=\cdots=\operatorname{inv}_{i}(\sigma)=0$. By definitions, we have $x_{k-1}=\cdots=x_{i-1}$ which proves that $\varphi$ is well defined. To prove the map $\varphi$ is a bijection, note that each permutation $\sigma$ can be uniquely recovered from its inversion vector $\left(\operatorname{inv}_{1}(\sigma), \ldots, \operatorname{inv}_{n+1}(\sigma)\right)$. Now we construct an inverse map $\psi: \Omega_{n} \rightarrow \mathcal{S}_{n+1}(321)$ of $\varphi$ recursively as follows. Given $x=$ $\left(x_{1}, \ldots, x_{n}\right)$, define $\psi(x)=\sigma=\sigma_{1} \cdots \sigma_{n+1}$ such that $\operatorname{inv}_{1}(\sigma)=0$ and for $2 \leqslant k \leqslant n+1$,

$$
\operatorname{inv}_{k}(\sigma)=\left\{\begin{array}{cc}
0 & \text { if } x_{k-1}=x_{k-2} \\
k-x_{k-1} & \text { otherwise }
\end{array}\right.
$$

It is not difficult to see that both $\psi \circ \varphi$ and $\varphi \circ \psi$ are identity map, i.e., $\varphi$ is a bijection. This completes the proof. 


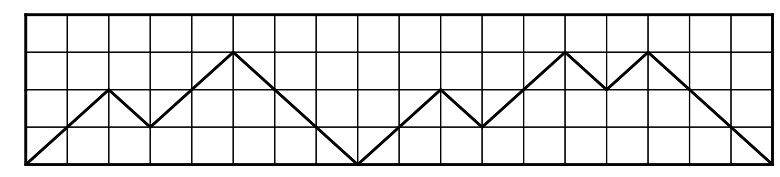

Figure 2: $x=(0,1,1,4,4,5,5,6) \mapsto D=$ uuduuddduuduududdd

When $n=3$, the inversion polynomial of $\mathcal{S}_{n}(321)$ is $I_{3}(321, q)=q^{4}+4 q^{3}+5 q^{2}+3 q+1$. Below is the list of the bijection $\varphi$,

$$
\begin{aligned}
q^{0}: & \{1234\} \stackrel{\varphi}{\longrightarrow}\{(0,0,0)\} ; \\
q^{1}: & \{1243,2134,1324\} \stackrel{\varphi}{\longrightarrow}\{(0,0,3),(1,1,1),(0,2,2)\} ; \\
q^{2}: & \{1342,1423,2143,2314,3124\} \stackrel{\varphi}{\longrightarrow}\{(0,2,3),(0,0,2),(1,1,3),(1,2,2),(0,1,1)\} ; \\
q^{3}: & \{2341,2413,3142,4123\} \stackrel{\varphi}{\longrightarrow}\{(1,2,3),(1,1,2),(0,1,3),(0,0,1)\} ; \\
q^{4}: & \{3412\} \stackrel{\varphi}{\longrightarrow}\{(0,1,2)\} .
\end{aligned}
$$

A Dyck path $D$ is a lattice path from $(0,0)$ to $(2 n, 0)$ in the $(x, y)$-plane with up-steps $(1,1)$ (abbreviated as ' $u$ ') and down-steps $(1,-1)$ (abbreviated as ' $d$ '), such that $D$ never falls below the $x$-axis. A valley $d u$ of the Dyck path $D$ is a down-step followed by an up-step. The height of a valley is defined to be the $y$-coordinate of its bottom. Denote by $\mathcal{D}_{n}$ the set of all Dyck paths of length $2 n$. Several bijections between $\mathcal{S}_{n}(321)$ and $\mathcal{D}_{n}$ have been established in the literature, see $[4,7,10,11,14,15,17,18,19,21,22]$. Here we will give a new bijection obtained easily from the above theorem. Morever, this bijection will allow to read the inversion number of a permutation as the sum of all valley heights and the number of valleys in the corresponding Dyck path.

Indeed, for $x=\left(x_{1}, \ldots, x_{n}\right) \in \Omega_{n}$, assuming $x_{0}=0$ and $x_{n+1}=n+1$, we construct a Dyck path $D_{x}$ as follows. By reading $i$ from 1 to $n+1$, for each $i$ we add an up-step and $x_{i}-x_{i-1}$ down-steps from left to right. Figure. 2 presents an example. It is obvious that this construction gives an bijection from $\Omega_{n+1}$ to $\mathcal{D}_{n+1}$.

If all valleys of a Dyck path $D$ have heights $a_{1}, \ldots, a_{k}$, denote by

$$
v(D)=\sum_{i=1}^{k}\left(a_{i}+1\right)
$$

Combining with Theorem 4, we can easily obtain our first main result.

Theorem 5. The map $\sigma \rightarrow D_{\varphi(\sigma)}$ is a bijection from $\mathcal{S}_{n+1}(321)$ to $\mathcal{D}_{n+1}$ such that

$$
\operatorname{inv}(\sigma)=v\left(D_{\varphi(\sigma)}\right)
$$

where $\varphi$ is defined in (2).

As an application of Theorem 5, we will give a counting formula on the number of 321 -avoiding permutations with a fixed inversion number. For any $D \in \mathcal{D}_{n}$, we define a 
tunnel of $D$ to be a horizontal segment between two lattice points of $D$ that intersects $D$ only in these two points, and stays always below $D$. From Theorem 5 , for $m \geqslant 0$, there is a bijection

$$
\mathcal{S}_{n, m}(321):=\left\{\sigma \in \mathcal{S}_{n}(321): i n v(\sigma)=m\right\} \longrightarrow \mathcal{D}_{n, m}:=\left\{D \in \mathcal{D}_{n}: v(D)=m\right\} .
$$

Theorem 6. For every $m \geqslant 0$,

$$
\left|\mathcal{S}_{n, m}(321)\right|=\sum_{b \vdash m}\left(\begin{array}{c}
n-\frac{\Delta(b)}{2} \\
l(b)
\end{array}\right) .
$$

where the sum runs over all compositions $b=\left(b_{1}, b_{2}, \ldots, b_{k}\right)$ of $m$, denoted $b \vdash m$, i.e.,

$$
m=b_{1}+b_{2}+\cdots+b_{k} \text { and } b_{i} \geqslant 1
$$

$l(b)=k$ is the length of $b$, and $\Delta(b):=\left|b_{1}\right|+\left|b_{2}-b_{1}\right|+\cdots+\left|b_{k}-b_{k-1}\right|+\left|b_{k}\right|$.

Proof. It is sufficient to consider $\left|\mathcal{D}_{n, m}\right|$. For any $D \in \mathcal{D}_{n, m}$, suppose that $D$ has $k$ valleys with heights $a_{1}, a_{2}, \cdots, a_{k}$, then $m=v(D)=\sum_{i=1}^{k}\left(a_{i}+1\right)$. Let $l_{i}$ be the length of the path $D$ located between the $i$-th and $(i+1)$-th valley, for $i=0,1,2, \cdots, k$. Then we have

$$
l_{i}=\left|a_{i+1}-a_{i}\right|+2 t_{i}, \quad \sum_{i=0}^{k} l_{i}=2 n, \quad t_{i} \geqslant 1 .
$$

Where $t_{i}$ is the number of tunnels between the $i$-th and $(i+1)$-th valley. Let $a_{0}=0$ and $a_{k+1}=0$ be the heights of the starting point and the terminal point of the Dyck path $D$, respectively. Write

$$
\Delta(a)=\sum_{i=0}^{k}\left|a_{i+1}-a_{i}\right|
$$

Then

$$
\begin{aligned}
& \#\left\{D \in \mathcal{D}_{n} \mid \text { all valleys of } D \text { have heights } a_{1}, a_{2}, \ldots, a_{k}\right\} \\
= & \#\left\{\left(l_{0}, l_{1}, \cdots, l_{k}\right)\left|l_{i}=\right| a_{i+1}-a_{i} \mid+2 t_{i}, \sum_{i=0}^{k} l_{i}=2 n, t_{i} \geqslant 1\right\} . \\
= & \#\left\{\left(t_{0}, t_{1}, \cdots, t_{k}\right) \mid t_{0}+t_{1}+\cdots+t_{k}=n-\frac{\Delta(a)}{2}, t_{i} \geqslant 1\right\} . \\
= & \left(\begin{array}{c}
n-\frac{\Delta(a)}{2}-1 \\
k
\end{array}\right)
\end{aligned}
$$

So we have

$$
\left|\mathcal{D}_{n, m}\right|=\sum_{\substack{\left(a_{1}+1\right)+\left(a_{2}+1\right)+\ldots+\left(a_{k}+1\right)=m \\
a_{i}+1 \geqslant 1}}\left(\begin{array}{c}
n-\frac{\Delta(a)}{2}-1 \\
k
\end{array}\right)
$$


Let $b_{i}=a_{i}+1$ for $1 \leqslant i \leqslant k, \quad b_{0}=b_{k+1}=0$, obviously $\Delta(b)=\sum_{i=0}^{k}\left|b_{i+1}-b_{i}\right|=\Delta(a)+2$. Hence

$$
\left|\mathcal{D}_{n, m}\right|=\sum_{\substack{b_{1}+b_{2}+\cdots+b_{k}=m \\
b_{i} \geqslant 1}}\left(\begin{array}{c}
n-\frac{\Delta(b)}{2} \\
k
\end{array}\right) .
$$

\section{Acknowledgements}

We would like to express our gratitude to the anonymous referee for many useful comments.

\section{References}

[1] J. Bandlow, Eric S. Egge, and K. Killpatrick. A weight-preserving bijection between Schröer paths and Schröer permutations. Ann. Comb., 6(3-4): 235-248, 2002.

[2] M. Barnabei, F. Bonetti, S. Elizalde, and M. Silimbani. Descent sets on 321-avoiding involutions and hook decompositions of partitions. J. Combin. Theory Ser. A, 128:132-148, 2014.

[3] Andrew M. Baxter. Refining enumeration schemes to count according to the inversion number. Pure Math. Appl. (PU. M. A.), 21(2): 137-160, 2010.

[4] S. C. Billey, W. Jockusch, and R. P. Stanley. Some combinatorial properties of Schubert polynomials. J. Algebraic Combin., 2(4): 345-374, 1993.

[5] William Y. C. Chen, Yu-Ping Deng, and Laura L.M. Yang. Motzkin paths and reduced decompositions for permutations with forbidden patterns. Electron. J. Combin., 9(2), \#R15, 2003.

[6] S. E. Cheng, S. Elizalde, A. Kasraouic, and B. E. Sagan. Inversion polynomials for 321-avoiding permutations. Discrete Math., 313(22):2552-2565, 2013

[7] A. Claesson and S. Kitaev. Classification of bijections between 321- and 132-avoiding permutations. Sém. Lothar. Combin. 60, Art. B60d, 30 pp, 2008.

[8] T. Dokos, T. Dwyer, B. P. Johnson, B. E. Sagan, and K. Selsor. Permutation patterns and statistics. Discrete Math., 312(18):2760-2775, 2012.

[9] Eric S. Egge. Restricted 3412-avoiding involutions, continued fractions, and Chebyshev polynomials. Adv. in Appl. Math., 33(3):451-475, 2004.

[10] S. Elizalde and E. Deutsch. A Simple and Unusual Bijection for Dyck Paths and its Consequences. Ann. Comb., 7(3):281-297, 2003.

[11] S. Elizalde and I. Pak. Bijections for refined restricted permutations. J. Combin. Theory Ser. A, 105(2):207-219, 2004.

[12] D. E. Knuth. The Art of Computer Programming I: Fundamental Algorithms, Addison-Wesley, Publishing Co., Reading, Mass.-London-Don Mills, Ont, 1969. 
[13] D. E. Knuth. The Art of Computer Programming III: Sorting and Searching, Addison-Wesley, Reading, MA, 1973.

[14] C. Krattenthaler. Permutations with restricted patterns and Dyck paths. Adv. in Appl. Math., 27(2-3):510-530, 2001.

[15] T. Mansour, Eva Y. D. Deng, and Rosena R. X. Du. Dyck paths and restricted permutations. Discrete Appl. Math., 154(11):1593-1605, 2006.

[16] S. Min and S. Park. The maximal-inversion statistic and pattern-avoiding permutations. Discrete Math., 309(9):2649-2657, 2009.

[17] A. Reifegerste. A generalization of Simion-Schmidt's bijection for restricted permutations. Electron. J. Combin., 9(2), \#R14, 2003.

[18] D. Richards. Ballot sequences and restricted permutations. Ars Combin., 25:83-86, 1988.

[19] D. Rotem. On a correspondence between binary trees and a certain type of permutations. 4(3):58-61, 1975.

[20] B. E. Sagan and C. D. Savage. Mahonian pairs. J. Combin. Theory Ser. A, 119(3):526-545, 2012.

[21] R. Simion and F. W. Schmidt. Restricted Permutations. European J. Combin., 6(4):383-406, 1985.

[22] J. West. Generating trees and the Catalan and Schröder numbers. Discrete Math., 146(1-3):247-262, 1995. 